\title{
The effects of Jerusalem artichoke and fermented soybean powder mixture supplementation on blood glucose and oxidative stress in subjects with prediabetes or newly diagnosed type 2 diabetes
}

Hyeon Yeong Ahn', Minjoo Kim', Cho Rong Seo ${ }^{2}$, Hye Jin Yoo ${ }^{3}$, Sang-Hyun Lee ${ }^{4}$ and Jong Ho Lee $\mathbb{C}^{1,3,5,6}$

\begin{abstract}
Background/Objectives: The objective of this study was to evaluate the effect of supplementation with a Jerusalem artichoke and fermented soybean powder mixture on blood glucose and oxidative stress levels.

Subjects/Methods: This randomized, double-blinded, placebo-controlled study was conducted on 60 subjects with impaired fasting glucose (IFG), impaired glucose tolerance (IGT), or newly diagnosed type 2 diabetes. The subjects were randomly assigned to either a group that ingested $40 \mathrm{~g}$ of a Jerusalem artichoke and fermented soybean powder mixture (19.45 g each) daily or a group that received a placebo for 12 weeks. Paired $t$-test and independent $t$-test were performed for comparisons within groups and between groups, respectively.

Results: Supplementation with the Jerusalem artichoke and fermented soybean powder mixture reduced the levels of fasting glucose $(p<0.001)$ and FFAs $(p=0.034)$, glucose at $60 \mathrm{~min}(p=0.004)$, glucose $(p=0.006)$ areas under the response curve $(A \cup C)$, homeostasis model assessment-insulin resistance $(p=0.018)$, and the urinary 8 -epiprostaglandin $F_{2 a}$ (8-epi-PGF $F_{2 a}$ ) level $(p=0.028)$. The changes $(\Delta)$ in urinary 8-epi-PGF $F_{2 a}$ glucose at $60 \mathrm{~min}, 120 \mathrm{~min}$, and AUC, FFAs at 0 min and AUC were significantly different between the two groups. In addition, $\triangle$ glucose at $120 \mathrm{~min}(r=0.472, p=0.027)$ and the $\triangle$ glucose AUC $(r=0.572, p=0.005)$ were positively correlated with $\triangle$ plasma malondialdehyde in the test group.

Conclusions: The consumption of a Jerusalem artichoke and fermented soybean powder mixture for 12 weeks was effective for reducing postprandial glucose and oxidative stress level, particularly 8-epi-PGF ${ }_{2 a}$, in subjects with IFG, IGT, or newly diagnosed type 2 diabetes.
\end{abstract}

Correspondence: Jong Ho. Lee (jhleeb@yonsei.ac.kr)

${ }^{1}$ Research Center for Silver Science, Institute of Symbiotic Life-TECH, Yonsei University, Seoul, Korea

${ }^{2}$ Department of Science for Aging, Graduate School of Yonsei University, Seoul, Korea

Full list of author information is available at the end of the article.

\section{Introduction}

Diabetes is a chronic metabolic disease that is common worldwide and is caused by reduced organ function, which results in insulin resistance (IR) or lack of insulin ${ }^{1}$. According to the World Health Organization, 347 million people worldwide had diabetes in 2013, and diabetes will

\section{(c) The Author(s) 2018}

(c) Open Access This article is licensed under a Creative Commons Attribution 4.0 International License, which permits use, sharing, adaptation, distribution and reproduction c. in any medium or format, as long as you give appropriate credit to the original author(s) and the source, provide a link to the Creative Commons license, and indicate if changes were made. The images or other third party material in this article are included in the article's Creative Commons license, unless indicated otherwise in a credit line to the material. If material is not included in the article's Creative Commons license and your intended use is not permitted by statutory regulation or exceeds the permitted use, you will need to obtain permission directly from the copyright holder. To view a copy of this license, visit http://creativecommons.org/licenses/by/4.0/. 
be the 7 th most common cause of death by $2030^{2}$. The prevalence of diabetes worldwide was $6.4 \%$ in 2010 and will increase to $7.7 \%$ by $2030^{3}$. According to the 2013 Korean National Health and Nutrition Examination Survey, the incidence of diabetes has increased $2 \%$ since $2012^{4}$. People with impaired fasting glucose (IFG), impaired glucose tolerance (IGT), or both are at high risk of progressing to type 2 diabetes, which is a risk factor for cardiovascular disease $^{5,6}$. The progression of IFG or IGT to type 2 diabetes can be delayed or prevented by lifestyle changes and pharmacological interventions ${ }^{7}$. According to the American Diabetes Association, medical nutrition therapy is an effective method for the management of diabetes ${ }^{8}$.

Jerusalem artichoke is a root vegetable, and the main components include fructans (fructose molecules connected by $\beta-2,1$ bonds), specifically inulin and fructooligosaccharides ${ }^{9}$. Additionally, chicory is a main source of inulin-type fructans, which are commonly extracted from the Compositae family and are known as prebiotic dietary fibers. Inulin-type fructans resist hydrolysis by human small intestinal digestive enzymes due to the $\beta$ configuration of the anomeric $\mathrm{C}_{2}$ in its fructose monomer; thus, inulin-type fructans have been classified as non-digestible oligosaccharides ${ }^{10,11}$. The enhancement of gastrointestinal functions is a primary endpoint that benefits from inulin-type fructans and reduces gastrointestinal-related disease risks, such as intestinal infections, colon cancer, and obesity ${ }^{12,13}$. Moreover, inulin and fructooligosaccharides stimulate the immune system, reduce the synthesis of triglycerides and fatty acids in the liver, and decrease blood glucose levels ${ }^{14}$. Several studies revealed that inulin-type fructans supplementation modulate glycemic indices, lipid profile, antioxidant status ${ }^{15}$, and some inflammatory, immunologic markers ${ }^{16}$, and calcium homeostasis ${ }^{17}$ in type 2 diabetes patients. Fermented soybeans have antidiabetic effects in diabetic animals and humans ${ }^{18-20}$. A previous study reported that the combination of Jerusalem artichoke and fermented soy had complementary antidiabetic effects through the potentiation of insulinotropic action and the reduction of IR in diabetic rats ${ }^{21}$. Therefore, we hypothesized that these findings might be extrapolated to humans, and the aim of this study was to investigate the effect of Jerusalem artichoke and fermented soybean powder mixture supplementation on blood glucose and oxidative stress levels.

\section{Subjects and methods Study subjects}

The study subjects were recruited from the outpatient clinics and the Health Service Center at the National Health Insurance Corporation Ilsan Hospital (Goyang, Korea) from June 2013 to December 2014. According to the glucose test results, 60 subjects with IFG $(100 \mathrm{mg} /$
$\mathrm{dL} \leq$ fasting blood glucose $\leq 125 \mathrm{mg} / \mathrm{dL}$ ), IGT [140 mg/ $\mathrm{dL} \leq 2 \mathrm{~h}$ blood glucose in the 75 -g oral glucose tolerance test $(\mathrm{OGTT}) \leq 199 \mathrm{mg} / \mathrm{dL}$ ], or newly diagnosed type 2 diabetes were enrolled in this study. The aim of the study was carefully explained to all participants who provided written informed consent. Subjects were excluded if they had any diagnosis of cancer, renal disease, liver disease, chronic gastrointestinal disorder, or chronic alcoholism; were pregnant or breastfeeding; or had taken insulin agents or glucose-lowering medications for the past month. The protocol was approved by the Institutional Review Board of Yonsei University and National Health Insurance Corporation Ilsan Hospital and registered at http://www.clinicaltrials.gov (NCT02506582). Study subjects were randomly assigned to the test or placebo group. A computer-generated block randomization program assigned the groups (test group:placebo group $=1: 1$ ). The study sample size was determined using SAS 9.4 software (SAS Institute Inc., NC, USA). In an exploratory clinical trial, the postprandial glucose (PPG) level at $30 \mathrm{~min}$ decreased by $10.0 \mathrm{mg} / \mathrm{dL}$ in the test group compared with a reduction of $2.5 \mathrm{mg} / \mathrm{dL}$ in the placebo group. The sample size for this study was determined using the following parameters:

(1) Superiority test

(2) Level of significance, $\alpha=0.05$

(3) $\beta=0.2$, power $=80 \%$

(4) Rates for participants in the test group and placebo group, $\lambda=1, n_{t}$ (participant number of the test group $)=\lambda \mathrm{n}_{\mathrm{c}}$ (participant number of the placebo group).

(5) We hypothesized that the changes in PPG levels at $30 \mathrm{~min}$ for the test group $\left(\mu_{\mathrm{t}}\right)$ would be $10.0 \mathrm{mg} / \mathrm{dL}$, and those changes for the placebo group $\left(\mu_{\mathrm{c}}\right)$ would be $2.5 \mathrm{mg} / \mathrm{dL}$.

(6) We assumed a standard deviation of $9.63 \mathrm{mg} / \mathrm{dL}$ for both the test group $\left(\sigma_{\mathrm{t}}\right)$ and the placebo group $\left(\sigma_{\mathrm{c}}\right)$.

$$
H_{0}: \mu_{t} \leq \mu_{c} \quad H_{1}: \mu_{t}>\mu_{c}
$$

The sample size was 21 subjects per group. Assuming a dropout rate of $30 \%$, we determined that 30 subjects should be recruited per group. Sixty subjects were enrolled, of whom eight dropped out after randomization due to personal reasons. Among 52 subjects who completed participation in the 12-week intervention study, five subjects who had less than $80 \%$ compliance were excluded from final analysis; the compliance was calculated as follows: Compliance $(\%)=$ Actual number of consumed supplements/Expected number of consumed supplements $\times 100$. Finally, 47 subjects [placebo group $(n=25)$, test group $(n=22)]$ were included in the final analysis (Fig. 1). 


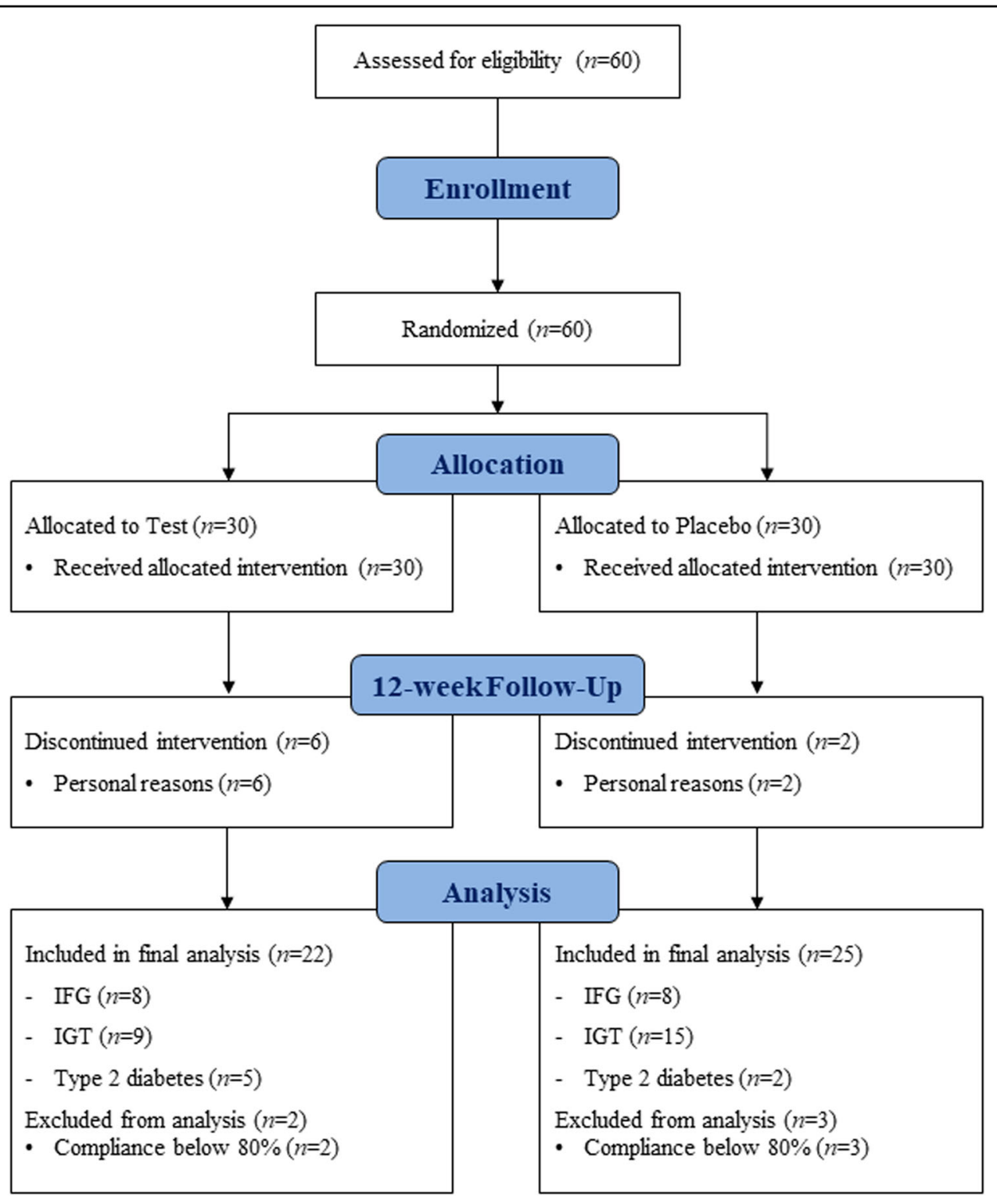

Fig. 1 Flow diagram demonstrating the process for inclusion and analysis of randomized clinical trials

\section{Study design and materials}

After recruiting the study subjects, the entire study process was conducted at the Clinical Nutrigenetics/ Nutrigenomics Lab in Yonsei University, Seoul, Korea. The study was a randomized, double-blinded, placebocontrolled trial. Subjects were divided into two groups. The test group consumed $40 \mathrm{~g}$ of a Jerusalem artichoke and fermented soybean powder mixture (3 times/day for 12 weeks) before each meal at home, and the placebo group consumed powdered rice flour (3 times/day for 12 weeks) before each meal at home; both the test and placebo products (powder) had low solubility, thus, we recommended the study subjects to take the powder first and then swallow it with water. The randomization list was blinded until the statistical analysis. The Jerusalem artichokes were sliced and steamed at $90-100^{\circ} \mathrm{C}$ for $30 \mathrm{~min}$, hot-air dried at $80^{\circ} \mathrm{C}$ for $24 \mathrm{~h}$ and powdered. The soybeans were fermented with Bacillus spp. at $42^{\circ} \mathrm{C}$ for $46 \mathrm{~h}$, freezedried, and powdered. The $40 \mathrm{~g}$ Jerusalem artichoke and fermented soybean powder mixture (19.45 g each) contained $150 \mathrm{mg} / \mathrm{g}$ of inulin, which consisted of $29 \%$ fructooligosaccharides. The test product contained the following components: $97.27 \%$ Jerusalem artichoke and fermented soybean powder, $2.65 \%$ cornstarch, $0.07 \%$ caramel coloring, and $0.01 \%$ L-menthol. The placebo product contained the following components: $84.71 \%$ rice flour, 
2.32\% cornstarch, $0.003 \%$ caramel coloring, $0.01 \%$ L-menthol, and $12.957 \%$ water. The products were provided by Midari Farm (Yeongwol, Kangwon-Do, Korea).

\section{Anthropometric parameters, blood pressure, and blood and urine collection}

All parameters were measured twice: at baseline (week 0 ) and follow-up (week 12). The body weight, height, and waist circumference were measured, and the body mass index (BMI) was determined as units of kilograms per square meter $\left(\mathrm{kg} / \mathrm{m}^{2}\right)$. Waist and hip circumferences were measured using a flexible measuring tape. Blood pressure (BP) was measured in the left arm with an automatic BP monitor (FT-200S, Jawon Medical, Gyeongsan, Korea). Blood and urine samples were also obtained at baseline and follow-up. After a 12-h fasting period, venous blood specimens were collected in ethylenediaminetetraacetate (EDTA)-treated tubes and plain tubes that were centrifuged to obtain plasma and serum. Urine was collected in polyethylene bottles containing $1 \%$ butylated hydroxytoluene. The collected samples were stored at $-80^{\circ} \mathrm{C}$ until analysis.

\section{OGTT and glucose-related biomarkers}

To conduct the OGTT (at baseline and follow-up, respectively), we prepared a glucose-water solution. Briefly, $75 \mathrm{~g}$ of anhydrous glucose was dissolved in $300 \mathrm{~mL}$ of room temperature water. The solution was consumed by the study participants after a 12-h overnight fast, and a fasting venous blood specimen was collected $(0 \mathrm{~min})$. Venous blood samples were also collected at 30 -min or 60-min intervals for $2 \mathrm{~h}$ (30,60, and $120 \mathrm{~min})$ in EDTAtreated tubes and plain tubes. Then, aliquots of the samples were stored at $-80^{\circ} \mathrm{C}$ prior to further analysis. Serum fasting glucose and blood glucose concentrations during the OGTT were measured using a hexokinase method with a Hitachi 7600 Autoanalyzer (Hitachi Ltd., Tokyo, Japan). Serum insulin was measured by an immunoradiometric assay kit from DIAsource ImmunoAssays S.A. (Louvain, Belgium). IR was calculated by the homeostasis model assessment (HOMA) method using the following equation: HOMA-IR $=[$ Fasting insulin $(\mu \mathrm{IU} / \mathrm{mL}) \times$ Fasting glucose $(\mathrm{mmol} / \mathrm{L})] / 22.5$. Serum Cpeptide was measured by an immunoradiometric assay with a C-peptide IRMA kit (Immunotech, Czech). Hemoglobin (HbA1c) was measured by an immunoturbidimetric analyzer with a turbidimeter.

\section{Serum lipid profile}

Free fatty acids (FFAs) were measured using an enzymatic assay using the acyl-CoA synthetase-acyl-CoA oxidase method with a Hitachi 7600 Autoanalyzer (Hitachi Ltd., Tokyo, Japan). Triglycerides (TGs) and total cholesterol were measured with a Hitachi 7600
Autoanalyzer (Hitachi Ltd., Tokyo, Japan). High-density lipoprotein (HDL) cholesterol was measured using an enzymatic method. Low-density lipoprotein (LDL) cholesterol was calculated by the Friedewald formula: LDL cholesterol $=$ total cholesterol $-[\mathrm{HDL}$ cholesterol $+(\mathrm{TG} / 5)]$.

\section{Urinary 8-epi-prostaglandin $\mathrm{F}_{2 a}$ and plasma malondialdehyde}

The urinary 8-epi-prostaglandin $\mathrm{F}_{2 \alpha}$ (8-epi-PGF $\mathrm{PG}_{2 \mathrm{a}}$ ) level was measured with a urinary isoprostane ELISA kit (Oxford Biomedical Research Inc., Rochester Hills, MI, USA). Urinary creatinine levels were determined using an alkaline picrate (Jaffe) reaction. Plasma malondialdehyde (MDA) was measured by determining the thiobarbituric acid-reactive substances (TBARS) with a TBARS Assay Kit (ZeptoMetrix Co., Buffalo, NY, USA).

\section{Dietary intake and total energy expenditure}

The subjects were instructed to maintain their eating habits and a normal level of physical activity during the study period to ensure that any changes that were observed were not due to diet or physical activity. Nutrient intake was mainly calculated based on the 3-day dietary records ( 2 weekdays and 1 weekend day) using the Computer Aided Nutritional Analysis Program (CAN-pro 3.0, Korean Nutrition Society, Seoul, Korea). This record was completed at home after the study subjects received detailed explanations from well-trained dietitians. This measurement was repeated at weeks 0 and 12 . In addition, the subjects completed a $24-\mathrm{h}$ recall and a semiquantitative food-frequency questionnaire with the assistance of a dietitian at weeks 0 and 12 to confirm the accuracy of the dietary record. Total energy expenditure (TEE) was calculated based on the basal metabolic rate (BMR), 24-h physical activity, and food-specific dynamic action, and the physical activity record was also completed at home on the same days that the dietary record was completed. The BMR was calculated using the Harris-Benedict equation. The 24-h physical activity was assessed by a physical activity questionnaire, developed based on the international physical activity questionnaire (IPAQ) and translated into Korean, at weeks 0 and 12; and all questions from the physical activity questionnaire were answered under the administration of interviewers to improve the accuracy of the survey.

\section{Primary and secondary outcomes}

The primary outcome of this study was an improvement in the 30 min PPG level during the OGTT. The secondary outcomes were an improvement in the fasting glucose level at the 12-week follow-up compared to baseline levels; differences in the blood glucose levels (subtract baseline values from follow-up values) between groups at 
each time point during the OGTT; and other glucoserelated biomarkers, including insulin, HOMA-IR, C-peptide, and HbA1c levels.

\section{Statistical analysis}

Statistical analysis was performed with SPSS version 21.0 (IBM, Chicago, IL, USA). A paired $t$-test was conducted to compare the effect of supplementation within each group both before and after intervention. An independent $t$-test was conducted to compare the net change $(\Delta$, difference from baseline) between the test and placebo groups. Pearson's correlation coefficient was used to examine relationships between variables. A logarithmic transformation was performed on skewed variables. The results are expressed as the mean \pm standard error (SE), and a $p$-value $<0.05$ was considered statistically significant.

\section{Results}

\section{Clinical and biochemical characteristics at baseline and} changes of dietary intake

The clinical and biochemical characteristics of the placebo and test groups are presented in Table 1. No significant differences in baseline characteristics [i.e., age, sex distribution, height, weight, BMI, waist-to-hip ratio (WHR), BPs, serum lipid profiles, HbA1c, HOMA-IR, C-peptide, 8-epi-PGF $F_{2 \alpha}$, or MDA] were observed between the groups. The urinary 8 -epi- $\mathrm{PGF}_{2 \alpha}$ level was significantly different between the two groups at baseline $(p=0.032)$. There were no significant differences observed in estimated total caloric intake (TCI), TEE, BMR, \% carbohydrate intake, $\%$ protein intake, and $\%$ fat intake, and cholesterol intake at baseline and 12-week follow-up period between the test and placebo group (Table S1). In addition, the changed values of dietary intake and TEE did not show any significances between the two groups.

\section{The effects of 12 weeks of supplementation with a Jerusalem artichoke and fermented soybean powder mixture on blood glucose levels and blood glucose-related biomarkers}

With the exception of BPs (Table 1), urinary 8-epiPGF $_{2 \alpha}$ levels (Fig. 2), blood glucose, and FFAs (Table 2), no significant mean changes in any of the tested clinical or biochemical characteristics were noted between the placebo and test groups. After 12 weeks of treatment, the test group exhibited significant reductions in blood glucose at $0 \mathrm{~min}(p=0.001)$ and $60 \mathrm{~min}(p=0.004)$ and glucose areas under the response curve (AUC) $(p=0.007)$ (Table 2). The $\Delta$ serum level of blood glucose at $60 \mathrm{~min}$ $(p=0.013)$ and $120 \mathrm{~min}(p=0.021)$ and the glucose AUC $(p=0.012)$ in the test group significantly differed from the placebo group, furthermore, these significances were maintained after adjusting for baseline values.
The 12-week intervention with Jerusalem artichoke and fermented soybean powder mixture supplementation resulted in a significant reduction in the HOMA-IR $(p=0.018)$ (Fig. 2). FFA serum levels at $0 \mathrm{~min}$ in the test group exhibited a significant decrease after the 12-week intervention $(p=0.034)$ (Table 2$)$. The $\Delta$ serum level of FFAs at 0 min $(p=0.008)$ and the FFA AUC $(p=0.044)$ in the test group significantly differed from the placebo group, furthermore, these significances were maintained after adjusting for baseline values. However, no significant differences in insulin levels at any time point and insulin AUC were noted between the placebo and test groups (Table 2).

\section{The effects of 12 weeks of supplementation with a Jerusalem artichoke and fermented soybean powder mixture on oxidative stress markers}

After 12 weeks of treatment, the test group exhibited a significant decrease in urinary 8-epi- $\mathrm{PGF}_{2 \alpha}$ levels $(p=0.028)$, and the placebo group exhibited a significant increase in the urinary 8-epi-PGF ${ }_{2 \alpha}$ level $(p=0.040)$ (Fig. 2). The $\Delta$ urinary 8 -epi-PGF $\mathrm{PF}_{2 \alpha}$ was significantly different between the two groups before $(p=0.003)$ and after adjusting for baseline value $(p=0.022)$. In the test group, the $\Delta$ glucose at $120 \mathrm{~min}$ was positively correlated with the $\Delta \mathrm{MDA}(r=0.472, p=0.027)$, and the $\Delta$ glucose AUC was positively correlated with the $\Delta$ MDA $(r=0.572, p=0.005)$ (Fig. 3). However, no significant associations were observed between $\Delta \mathrm{MDA}$ and $\Delta$ glucose $120 \mathrm{~min}$ or between $\Delta$ MDA and $\Delta$ glucose AUC in the placebo group.

\section{Discussion}

The antidiabetic effects of Jerusalem artichoke and fermented soybeans have raised interest in recent years; a previous study demonstrated that the combination of Jerusalem artichoke and fermented soybean had complementary antidiabetic effects through the potentiation of insulinotropic action and the reduction of IR in diabetic rats $^{21}$. Therefore, we hypothesized that these findings might be extrapolated to humans. This randomized, double-blind, placebo-controlled study evaluated the effect of supplementation with a Jerusalem artichoke and fermented soybean powder mixture on glucose control in subjects with IFG, IGT, or newly diagnosed type 2 diabetes. As a result, the primary outcome (PPG level at $30 \mathrm{~min}$; a time point when blood glucose levels increased promptly and rapidly in response to food intake) did not show any significant changes. However, changes in the PPG level at 60 and 120 min showed greater decrease in the test group than in the placebo group; finally, the glucose AUC was significantly reduced in the test group. Thus, overall glucose metabolism was improved, although the test product was not effective at slowing the prompt 


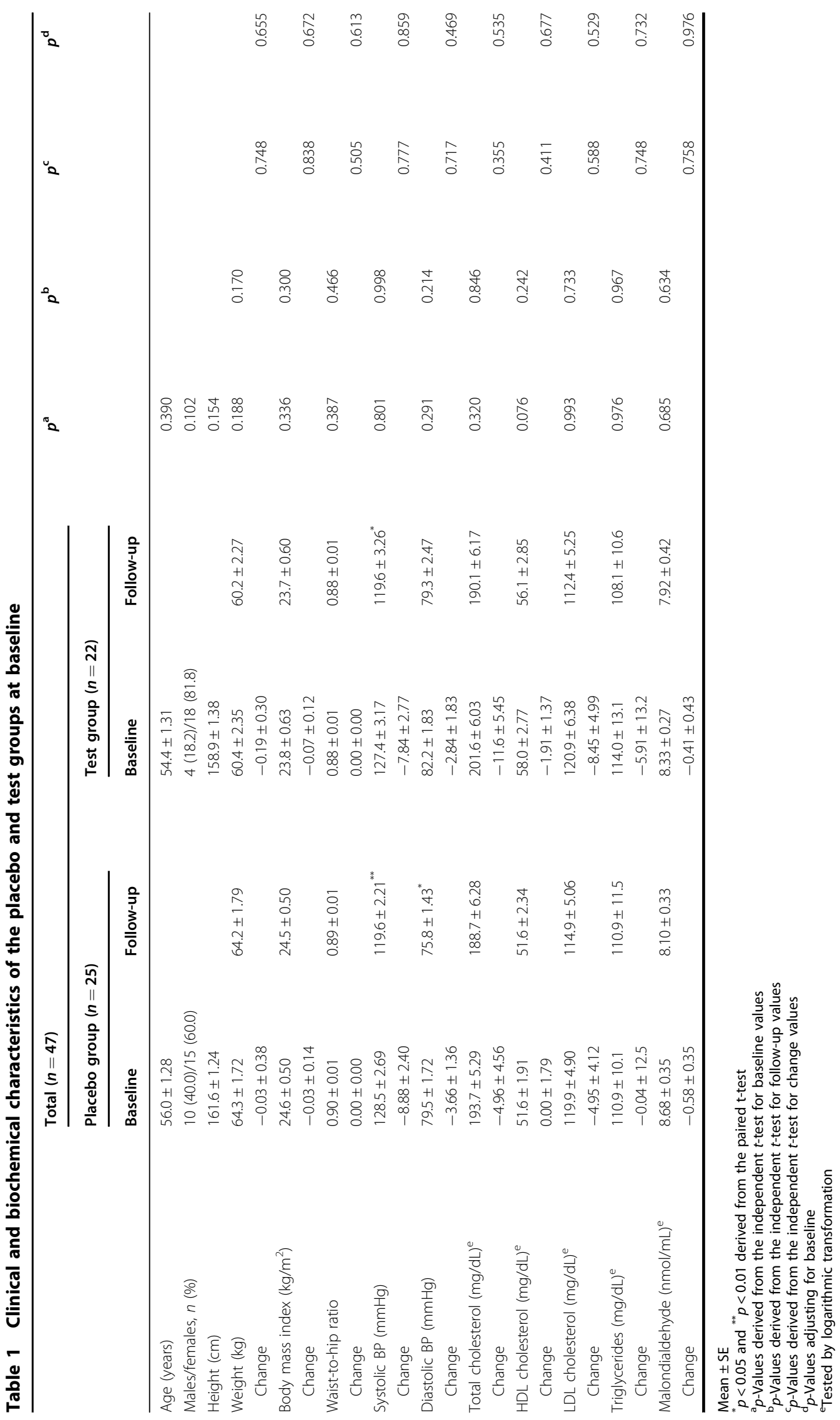


(a)

HOMA-IR

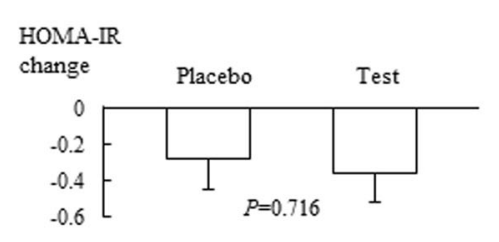

HOMA-IR

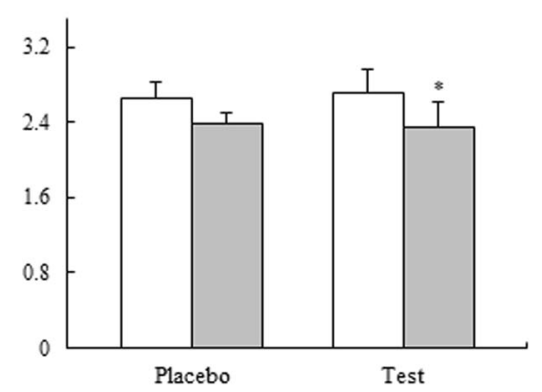

(b)

\section{Urinary 8-epi-PGF $2 a$}

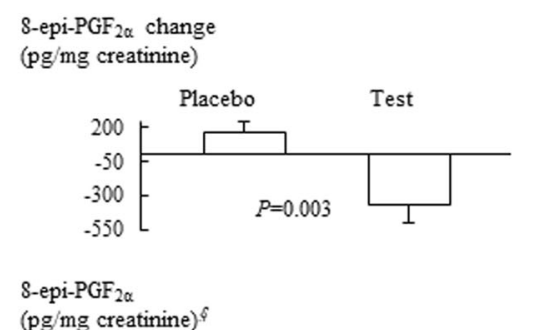

( $\mathrm{pg} / \mathrm{mg}$ creatinine $)^{4}$

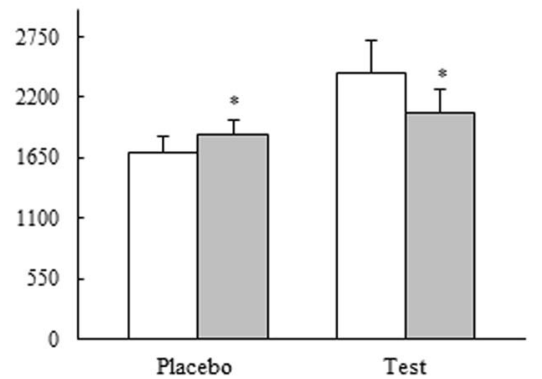

Fig. 2 HOMA-IR and urinary 8-epi-PGF $2 a$ levels at the initial visit $(\square)$ and the 12-week follow-up $(\square)$ and mean changes according to treatment. Mean $\pm \mathrm{SE}$. ${ }^{\varsigma}$ Tested by logarithmic transformation. $p$-Values were determined using the independent $t$-test. ${ }^{*} p<0.05$ compared with baseline values in each group as determined by the paired $t$-test

glucose absorption. Moreover, decreased blood glucose and HOMA-IR levels in the test group revealed the antidiabetic effects of Jerusalem artichoke and fermented soybeans in human subjects. In addition, diminished FFA levels and 8-epi-PGF ${ }_{2 \alpha}$ in the test group and the positive correlation between glucose and MDA levels suggested possible mechanisms related to the antidiabetic effects of Jerusalem artichoke and fermented soybeans.

The main component of Jerusalem artichoke is fructans, specifically inulin and fructooligosaccharides, which are dietary fiber that slow down absorption of blood glucose ${ }^{22}$ and are not absorbed in the intestines ${ }^{23}$. Therefore, consumption of Jerusalem artichoke did not affect insulin levels, and the present study revealed that supplementation with the Jerusalem artichoke and fermented soybean powder mixture did not affect insulin levels. Because of this absorption inhibition effect, we set a PPG level at 30 min, a time point that blood glucose increases rapidly, as a primary outcome rather than fasting glucose levels or PPG level at $120 \mathrm{~min}$. Indeed, Chamukuttan et al. ${ }^{24}$ demonstrated that elevated PPG level at 30 min during OGTT was independently associated with incident diabetes in Asian Indians population; and Kabaroğlu et al. ${ }^{25}$ indicated that increased PPG level at $30 \mathrm{~min}(>140 \mathrm{mg} /$ $\mathrm{dL}$ ) during OGTT was related to higher systemic inflammatory levels in obese adolescents with normal glucose intolerance (fasting glucose $<100 \mathrm{mg} / \mathrm{dL}$ and PPG level at $120 \mathrm{~min}<140 \mathrm{mg} / \mathrm{dL}$ ). Thus, not only fasting glucose or PPG level at $120 \mathrm{~min}$ but also PPG level at 30 min becomes an important factor for regulating the risk of diabetes or diabetic-related disease. Moreover, in Korea, inulin is approved as an official functional material by Ministry of Food and Drug Safety; it stipulates that inulin has a function on improvement of PPG level. Based on the evidences, we assessed $30 \mathrm{~min}$ glucose in OGTT as a primary outcome. In the present study, although significant changes were not observed in $30 \mathrm{~min}$ glucose, changes in the PPG level at 60 and $120 \mathrm{~min}$ were significantly decreased in the test group than in the placebo group. Furthermore, the glucose AUC showed significant reduction in the test group. Thus, overall glucose metabolism was improved. Several studies also reported a beneficial effect of inulin on fasting glycemic control. A previous study demonstrated that fructooligosaccharide supplementation reduced fasting plasma glucose levels after 2 weeks in type 2 diabetes patients ${ }^{26}$. Another clinical trial demonstrated an antidiabetic effect of inulinenriched pasta in humans, which reduced fasting glucose, HbA1c, and HOMA-IR levels ${ }^{27}$. In vivo studies have reported that Jerusalem artichoke supplementation improved IR and decreased the results of the OGTT. In addition, plasma glucose levels decreased with increasing levels of supplemented Jerusalem artichoke ${ }^{28,29}$. Inulin and fructooligosaccharides might affect glucose levels by changing the secretion of glucagon-like peptide-1, which increases insulin secretion, delays gastric emptying, 


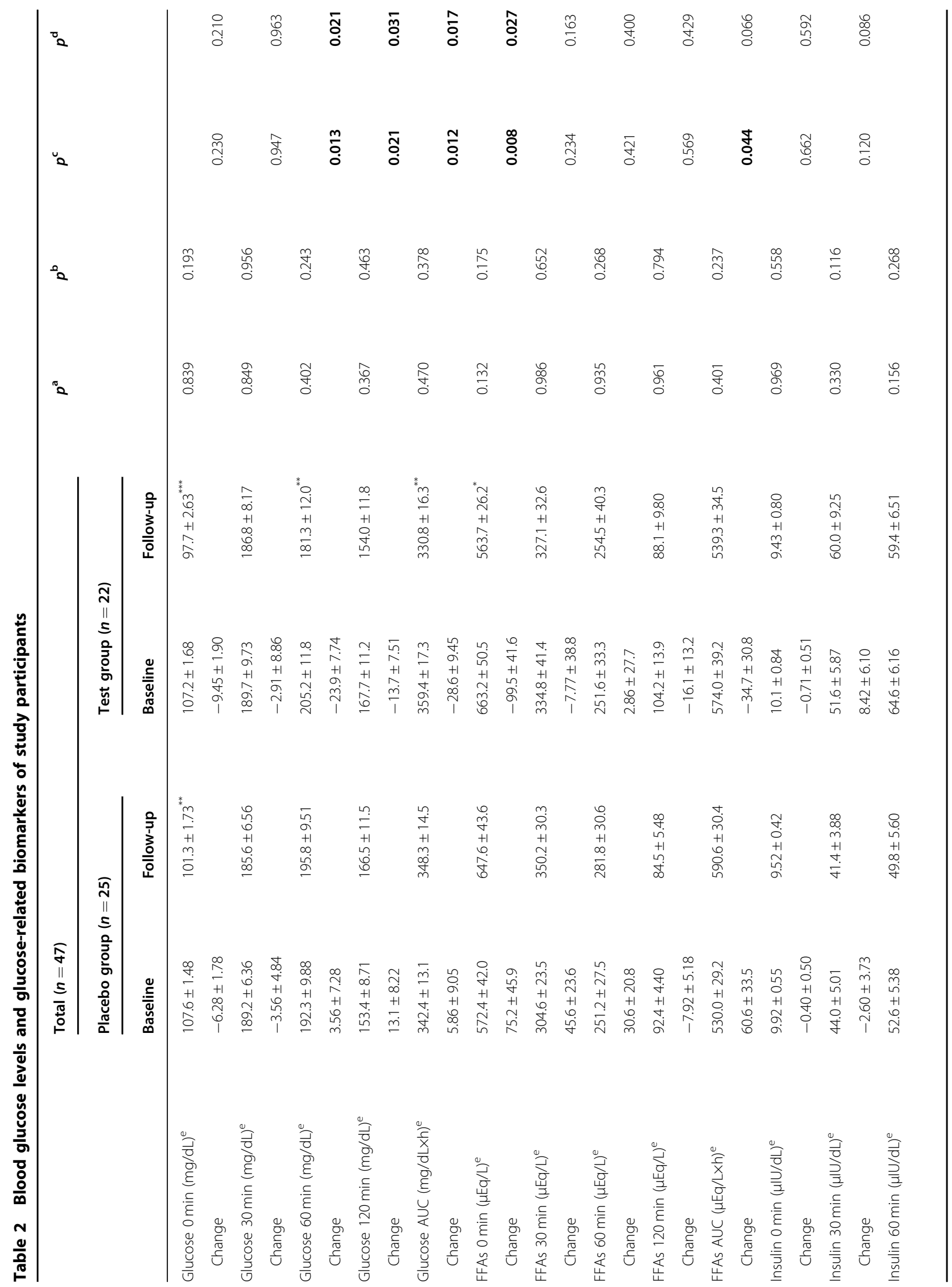




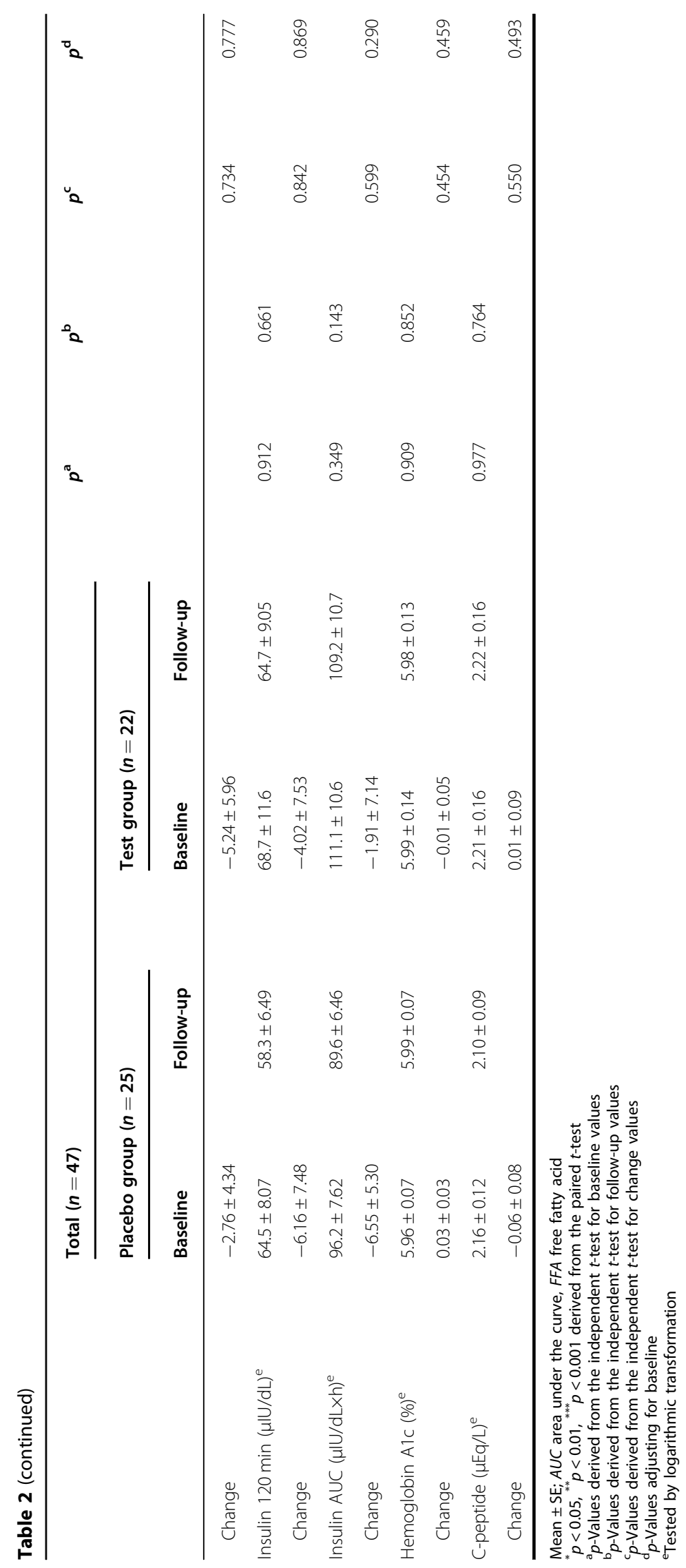



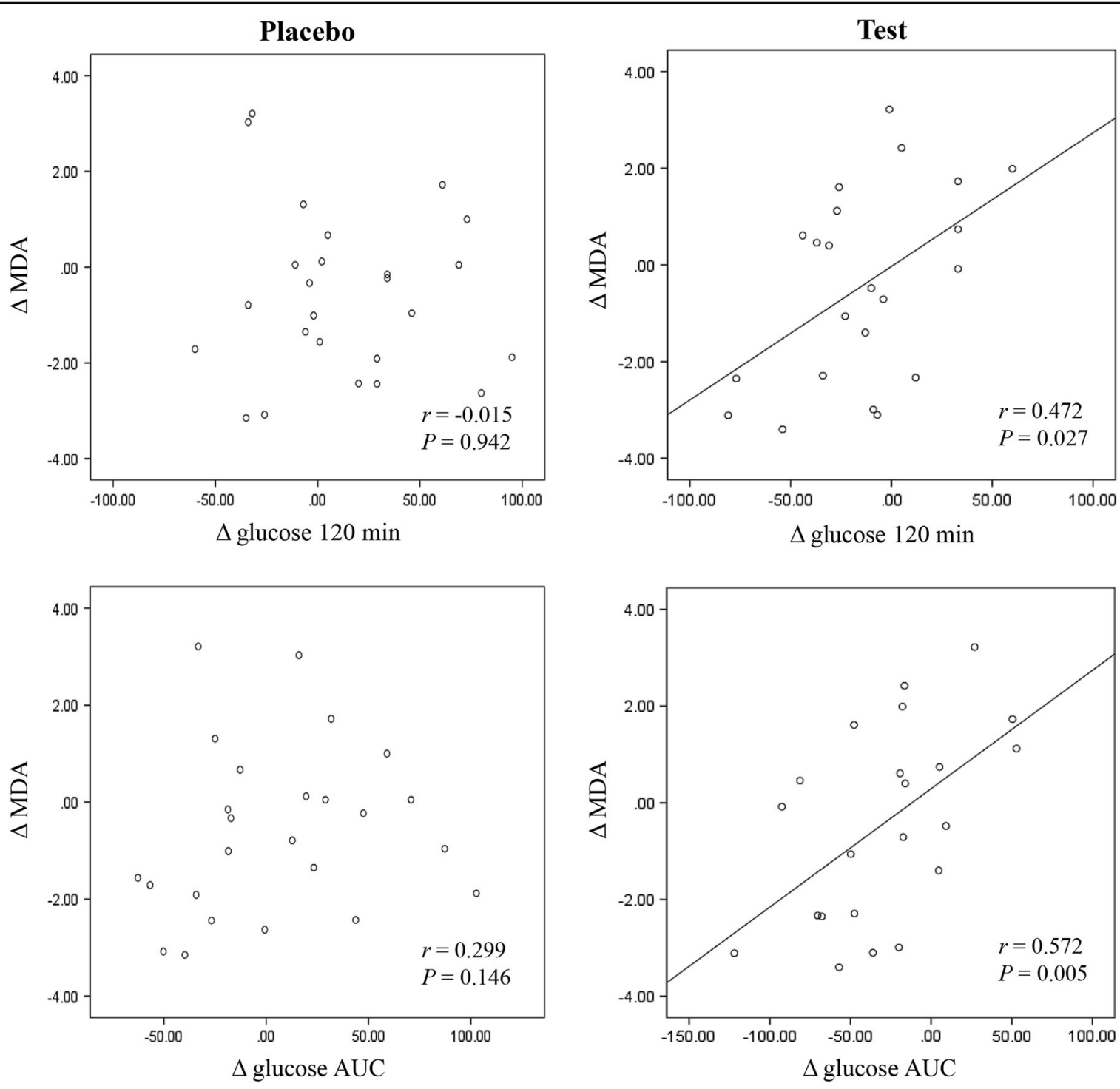

Fig. 3 Correlation between changes ( $\Delta$, difference from baseline) in blood glucose levels and plasma MDA in the placebo and test groups. $r$ Pearson's correlation coefficients

promotes $\beta$-cell proliferation, and reduces $\beta$-cell apopto$\mathrm{sis}^{30-34}$. The mechanism of the hypoglycemic effect of Jerusalem artichoke remains largely unknown.

In clinical trials, the consumption of an inulin-rich product reduced HbA1c levels over 5 weeks ${ }^{27}$, and the consumption of a fermented soybean product decreased fasting blood glucose and HbA1c levels after 3 months in subjects with borderline and mild type 2 diabetes ${ }^{35}$. However, in the present study, HbA1c levels did not change during the study period. According to several studies, HbA1c levels show a poor correlation with the prediabetes status ${ }^{36-38}$. Since most of our study subjects displayed IFG levels or IGT and only a few individuals with newly diagnosed type 2 diabetes were included in the study, HbA1c levels might not be a proper tool to evaluate the improvement in glucose control in the present study.

Previous studies have demonstrated that long-term consumption of fermented soybean attenuated IR and improved glucose homeostasis in a type 2 diabetic animal model $^{18,39}$. The biologically active components of fermented soybean include isoflavonoids and small peptides that are associated with IR and glycemic control ${ }^{40,41}$. These findings suggest potential mechanisms to explain the antidiabetic effect of fermented soybeans. According to a previous study, isoflavonoids might have an antidiabetic effect through estrogen-like action. Estrogen reduces IR through inhibition of intestinal glucose uptake and prevention of glucose-induced lipid peroxidation by 
inhibiting the sodium-dependent glucose transporter ${ }^{42}$. In addition, estrogen is associated with the stimulation of liver fatty acid metabolism and suppression of hepatic glucose production ${ }^{43}$. FFAs cause IR by inhibiting insulinstimulated glucose uptake. High levels of plasma FFAs are associated with an increase in diacylglycerol and activation of protein kinase $\mathrm{C}$, leading to increased tyrosine phosphorylation of insulin receptor substrate-1 and resulting in increased insulin-stimulated glucose transport activity and $\mathrm{IR}^{44,45}$. The present study demonstrated that supplementation with a Jerusalem artichoke and fermented soybean powder mixture decreased FFAs and IR, and both of these effects are explained by the presence of isoflavonoids in fermented soybeans.

Type 2 diabetes is associated with oxidative stress, and lipid peroxidation is the main marker of oxidative stress that plays a major role in the pathogenesis of type 2 diabetes $^{46-48}$. In addition, 8-epi-PGF $2 \alpha$ is a secondary end product of peroxidation that can impair $\beta$-cell function and lead to apoptosis ${ }^{49}$. Improved metabolic control reduced 8 -epi-PGF $\mathrm{PG}_{2 \alpha}$ by $32 \%$ in type 2 diabetes patients, and a clinical trial reported that 8-epi-PGF ${ }_{2 \alpha}$ was threefold higher in type 2 diabetes patients than in healthy individuals ${ }^{50,51}$. Several investigators reported that 8-epi$\mathrm{PGF}_{2 \alpha}$ is a predictor of glycemic control and oxidation status in patients with type 2 diabetes $^{52}$ and a reliable marker of IGT ${ }^{53}$. In the present study, we observed that 8epi-PGF $F_{2 \alpha}$ levels were significantly decreased in the test group. Jerusalem artichoke and fermented soybean powder mixture supplementation reduced oxidative stress in subjects with IFG, IGT, or newly diagnosed type 2 diabetes. Close interrelationships between changes of glucose and MDA were demonstrated in the present study despite the lack of differences in MDA levels between the placebo and test groups as well as no significant changes after the 12-week intervention. MDA is a primary biomarker of free radical-mediated lipid damage and oxidative stress and is elevated in type 2 diabetes patients ${ }^{54-56}$. This previous finding could explain the positive correlation between $\Delta$ glucose and $\Delta \mathrm{MDA}$ in the test group of this study, indicating that lipid peroxidation was related to glycemic control and was affected by Jerusalem artichoke and fermented soybean powder mixture supplementation. The present results indicate that inulin in Jerusalem artichoke and isoflavonoids in fermented soybeans might complementarily affect glucose control, and Jerusalem artichoke and fermented soybean powder mixture supplementation could ameliorate existing oxidative stress in subjects with IFG, IGT, or newly diagnosed type 2 diabetes.

There are several limitations of our study design. First, we specifically focused on Korean subjects with IFG, IGT, or newly diagnosed type 2 diabetes. Therefore, our data cannot be generalized to other ethnic groups or to severe type 2 diabetes patients. Second, the sample size is relatively small; thus, the results should be interpreted with caution. Third, the levels of any inflammatory, antioxidant, or HDL markers that may affect or be associated with the results of this study were not measured. Finally, we did not assess the dose-dependent effects of the test product; thus, the effects of various doses of the test product should be verified through further studies. Despite these limitations, Jerusalem artichoke and fermented soybean powder mixture supplementation exhibited antidiabetic and antioxidant effects. In conclusion, the consumption of a Jerusalem artichoke and fermented soybean powder mixture for 12 weeks was effective for reducing PPG and oxidative stress level, particularly 8 -epi-PGF $2 \alpha$, in subjects with IFG, IGT, or newly diagnosed type 2 diabetes.

\section{Acknowledgements \\ We thank all the volunteers who participated in this study for their cooperation. This work was supported in part by the "Food Functionality Evaluation Program" under the Ministry of Food, Agriculture, Forestry and Fisheries and by the Bio-Synergy Research Project (NRF-2012M3A9C4048762) of the Ministry of Science, ICT and Future Planning through the National Research Foundation, Republic of Korea.}

\section{Author details \\ ${ }^{1}$ Research Center for Silver Science, Institute of Symbiotic Life-TECH, Yonsei University, Seoul, Korea. ${ }^{2}$ Department of Science for Aging, Graduate School of Yonsei University, Seoul, Korea. ${ }^{3}$ Department of Food and Nutrition, Brain Korea 21 PLUS Project, College of Human Ecology, Yonsei University, Seoul, Korea. ${ }^{4}$ Department of Family Practice, National Health Insurance Corporation IIsan Hospital, Goyang, Korea. ${ }^{5}$ Department of Food and Nutrition, National Leading Research Laboratory of Clinical Nutrigenetics/Nutrigenomics, College of Human Ecology, Yonsei University, Seoul, Korea. ${ }^{6}$ Cardiovascular Research Institute, Yonsei University College of Medicine, Seoul, Korea}

Conflict of interest

The authors declare that they have no conflict of interest.

\section{Publisher's note}

Springer Nature remains neutral with regard to jurisdictional claims in published maps and institutional affiliations.

Supplementary Information accompanies this paper at (https://doi.org/ 10.1038/s41387-018-0052-y).

Received: 25 January 2018 Revised: 2 May 2018 Accepted: 31 May 2018 Published online: 19 July 2018

\section{References}

1. Cavaghan, M. K., Ehrmann, D. A. \& Polonsky, K. S. Interactions between insulin resistance and insulin secretion in the development of glucose intolerance. J. Clin. Invest. 106, 329-333 (2000).

2. Mathers, C. D. \& Loncar, D. Projections of global mortality and burden of disease from 2002 to 2030. PLoS Med. 3, e442 (2006).

3. Shaw, J. E., Sicree, R. A. \& Zimmet, P. Z. Global estimates of the prevalence of diabetes for 2010 and 2030. Diabetes Res. Clin. Pract. 87, 4-14 (2010).

4. Centers for Disease Control \& Prevention. Korea Health Statistics 2013: Korea National Health and Nutrition Examination Survey (KNHANESVI) (Ministry of Health and Welfare, Korea, 2013).

5. Muntner, P., He, J., Chen, J., Fonseca, V. \& Whelton, P. K. Prevalence of nontraditional cardiovascular disease risk factors among persons with impaired 
fasting glucose, impaired glucose tolerance, diabetes, and the metabolic syndrome: analysis of the third National Health and Nutrition Examination Survey (NHANES III). Ann. Epidemiol. 14, 686-695 (2004).

6. Henry, P., Thomas, F., Benetos, A. \& Guize, L. Impaired fasting glucose, blood pressure and cardiovascular disease mortality. Hypertension 40, 458-463 (2002).

7. Knowler, W. C. et al. Reduction in the incidence of type 2 diabetes with lifestyle intervention or metformin. N. Engl. J. Med. 346, 393-403 (2002).

8. Franz, M. J. et al. The evidence for medical nutrition therapy for type 1 and type 2 diabetes in adults. J. Am. Diet. Assoc. 110, 1852-1889 (2010).

9. Niness, K. R. Inulin and oligofructose: what are they? J. Nutr. 129, 1402S-1406S (1999).

10. Delzenne, N. M. \& Roberfroid, M. R. Physiological effects of non-digestible oligosaccharides. LWT - Food Sci. Technol. 27, 1-6 (1994).

11. Roberfroid, M. \& Slavin, J. Nondigestible oligosaccharides. Crit. Rev. Food Sci. Nutr. 40, 461-480 (2000)

12. Roberfroid, M. B. Inulin-type fructans: functional food ingredients. J. Nutr. 137, 2493S-2502S (2007).

13. Kolida, S. \& Gibson, G. R. Prebiotic capacity of inulin-type fructans. J. Nutr. 137, 2503S-2506S (2007).

14. Kaur, N. \& Gupta, A. K. Applications of inulin and oligofructose in health and nutrition. J. Biosci. 27, 703-714 (2002)

15. Aliasgharzadeh, A. et al. A combination of prebiotic inulin and oligofructose improve some of cardiovascular disease risk factors in women with type 2 diabetes: a randomized controlled clinical trial. Adv. Pharm. Bull. 5, 507-514 (2015).

16. Dehghan, P., Farhangi, M. A., Tavakoli, F., Aliasgarzadeh, A. \& Akbari, A. M. Impact of prebiotic supplementation on T-cell subsets and their related cytokines, anthropometric features and blood pressure in patients with type 2 diabetes mellitus: a randomized placebo-controlled trial. Complement. Ther. Med. 24, 96-102 (2016).

17. Farhangi, M. A., Javid, A. Z. \& Dehghan, P. The effect of enriched chicory inulin on liver enzymes, calcium homeostasis and hematological parameters in patients with type 2 diabetes mellitus: a randomized placebo-controlled trial. Prim. Care Diabetes 10, 265-271 (2016).

18. Kwon, D. Y., Hong, S. M., Lee, J. E., Sung, S. R. \& Park, S. Long-term consumption of fermented soybean-derived Chungkookjang attenuates hepatic insulin resistance in 90\% pancreatectomized diabetic rats. Horm. Metab. Res. 39 752-757 (2007)

19. Kwon, D. Y., Daily, J. W. 3rd, Kim, H. J. \& Park, S. Antidiabetic effects of fermented soybean products on type 2 diabetes. Nutr. Res. 30, 1-13 (2010).

20. Taniguchi, A. et al. Natto and viscous vegetables in a Japanese style meal suppress postprandial glucose and insulin responses. Asia Pac. J. Clin. Nutr. 17, 663-668 (2008)

21. Yang, $\mathrm{H}$. J. et al. Jerusalem artichoke and chungkookjang additively improve insulin secretion and sensitivity in diabetic rats. Nutr. Metab. (Lond.) 9, 112 (2012).

22. Ahmed, W. \& Rashid, S. Functional and therapeutic potential of inulin: a comprehensive review. Crit. Rev. Food Sci. Nutr. https://doi.org/10.1080/ 10408398.2017.1355775 (2017).

23. Rumessen, J. J., Bodé, S., Hamberg, O. \& Gudmand-Høyer, E. Fructans of Jerusalem artichokes: intestinal transport, absorption, fermentation, and influence on blood glucose, insulin, and C-peptide responses in healthy subjects. Am. J. Clin. Nutr. 52, 675-681 (1990).

24. Chamukuttan, S. et al. Baseline level of 30-min plasma glucose is an independent predictor of incident diabetes among Asian Indians: analysis of two diabetes prevention programmes. Diabetes Metab. Res. Rev. 32, 762-767 (2016).

25. Kabaroğlu, C. et al. Elevated glucose level at 30 min during an oral glucose tolerance test in obese adolescents: a new disorder of glucose tolerance. Endocr. J. 60, 197-205 (2013).

26. Yamashita, K., Kawai, K. \& Itakura, M. Effects of fructo-oligosaccharides on blood glucose and serum lipids in diabetic subjects. Nutr. Res. 4, 961-966 (1984).

27. Russo, F. et al. Metabolic effects of a diet with inulin-enriched pasta in healthy young volunteers. Curr. Pharm. Des. 16, 825-831 (2010).

28. Chang, W. C. et al. Beneficial effects of soluble dietary Jerusalem artichoke (Helianthus tuberosus) in the prevention of the onset of type 2 diabetes and non-alcoholic fatty liver disease in high-fructose diet-fed rats. Br. J. Nutr. 112, 709-717 (2014)

29. Samal, L., Chaturvedi, V. B., Saikumar, G., Somvanshi, R. \& Pattanaik, A. K. Prebiotic potential of Jerusalem artichoke (Helianthus tuberosus L.) in Wistar rats: effects of levels of supplementation on hindgut fermentation, intestinal morphology, blood metabolites and immune response. J. Sci. Food Agric. 95, 1689-1696 (2015).

30. Cani, P. D. \& Delzenne, N. M. The role of the gut microbiota in energy metabolism and metabolic disease. Curr. Pharm. Des. 15, 1546-1558 (2009).

31. Baggio, L. L. \& Drucker, D. J. Biology of incretins: GLP-1 and GIP. Gastroenterology 132, 2131-2157 (2007).

32. Nauck, M. A. et al. Normalization of fasting hyperglycaemia by exogenous glucagon-like peptide 1 (7-36 amide) in type 2 (non-insulin-dependent) diabetic patients. Diabetologia 36, 741-744 (1993).

33. Buteau, J., Foisy, S., Joly, E. \& Prentki, M. Glucagon-like peptide 1 induces pancreatic beta-cell proliferation via transactivation of the epidermal growth factor receptor. Diabetes 52, 124-132 (2003).

34. Farilla, L. et al. Glucagon-like peptide-1 promotes islet cell growth and inhibits apoptosis in Zucker diabetic rats. Endocrinology 143, 4397-4408 (2002).

35. Fujita, H., Yamagami, T. \& Ohshima, K. Long-term ingestion of a fermented soybean-derived Touchi-extract with alpha-glucosidase inhibitory activity is safe and effective in humans with borderline and mild type-2 diabetes. J. Nutr. 131, 2105-2108 (2001).

36. van't Riet, E. et al. Relationship between A1C and glucose levels in the general Dutch population: the new Hoorn study. Diabetes Care 33, 61-66 (2010).

37. Gosmanov, A. R. \& Wan, J. Low positive predictive value of hemoglobin A1C for diagnosis of prediabetes in clinical practice. Am. J. Med. Sci. 348, 191-194 (2014).

38. Guo, F., Moellering, D. R. \& Garvey, W. T. Use of HbA1c for diagnoses of diabetes and prediabetes: comparison with diagnoses based on fasting and 2hr glucose values and effects of gender, race, and age. Metab. Syndr. Relat. Disord. 12, 258-268 (2014).

39. Kwon, D. Y. et al. Long-term consumption of fermented soybean-derived Chungkookjang enhances insulinotropic action unlike soybeans in $90 \%$ pancreatectomized diabetic rats. Eur. J. Nutr. 46, 44-52 (2007).

40. Mezei, O. et al. Soy isoflavones exert antidiabetic and hypolipidemic effects through the PPAR pathways in obese Zucker rats and murine RAW 264.7 cells. J. Nutr. 133, 1238-1243 (2003).

41. Davis, J. et al. Soy protein influences the development of the metabolic syndrome in male obese ZDFxSHHF rats. Horm. Metab. Res. 37, 316-325 (2005).

42. Vedavanam, K., Srijayanta, S., O'Reilly, J., Raman, A. \& Wiseman, H. Antioxidant action and potential antidiabetic properties of an isoflavonoid-containing soyabean phytochemical extract (SPE). Phytother. Res. 13, 601-608 (1999).

43. Louet, J. F., LeMay, C. \& Mauvais-Jarvis, F. Antidiabetic actions of estrogen: insight from human and genetic mouse models. Curr. Atheroscler. Rep. 6 , 180-185 (2004).

44. Itani, S. I., Ruderman, N. B., Schmieder, F. \& Boden, G. Lipid-induced insulin resistance in human muscle is associated with changes in diacylglycerol, protein kinase C, and IkappaB-alpha. Diabetes 51, 2005-2011 (2002).

45. Yu, C. et al. Mechanism by which fatty acids inhibit insulin activation of insulin receptor substrate-1 (IRS-1)-associated phosphatidylinositol 3-kinase activity in muscle. J. Biol. Chem. 277, 50230-50236 (2002).

46. Evans, J. L., Goldfine, I. D., Maddux, B. A. \& Grodsky, G. M. Oxidative stress and stress-activated signaling pathways: a unifying hypothesis of type 2 diabetes. Endocr. Rev. 23, 599-622 (2002).

47. Rehman, K. \& Akash, M. S. H. Mechanism of generation of oxidative stress and pathophysiology of type 2 diabetes mellitus: how are they interlinked? J. Cell. Biochem. 118, 3577-3585 (2017).

48. Ramalingam, L. et al. The renin angiotensin system, oxidative stress and mitochondrial function in obesity and insulin resistance. Biochim. Biophys. Acta 1863, 1106-1114 (2017).

49. Miwa, I., Ichimura, N., Sugiura, M., Hamada, Y. \& Taniguchi, S. Inhibition of glucose-induced insulin secretion by 4-hydroxy-2-nonenal and other lipid peroxidation products. Endocrinology 141, 2767-2772 (2000).

50. Davì, G. et al. In vivo formation of 8-iso-prostaglandin f2alpha and platelet activation in diabetes mellitus: effects of improved metabolic control and vitamin E supplementation. Circulation 99, 224-229 (1999).

51. Gopaul, N. K. et al. Plasma 8-epi-PGF2 alpha levels are elevated in individuals with non-insulin dependent diabetes mellitus. FEBS Lett. 368, 225-229 (1995).

52. Nakhjavani, M. et al. Prostaglandin F2 alpha plasma concentration predicts glycemic control and oxidation status in patients with type 2 diabetes mellitus. Clin. Lab. 60, 2073-2080 (2014). 
53. Mure, K. et al. Urinary 8-iso-prostaglandin F2a as a marker of metabolic risks in the general Japanese population: the ROAD study. Obesity (Silver Spring) 23, 1517-1524 (2015).

54. Likidlilid, A., Patchanans, N., Peerapatdit, T. \& Sriratanasathavorn, C. Lipid peroxidation and antioxidant enzyme activities in erythrocytes of type 2 diabetic patients. J. Med. Assoc. Thai 93, 682-693 (2010).
55. Gallou, G. et al. Plasma malondialdehyde in type 1 and type 2 diabetic patients. Clin. Chim. Acta 214, 227-234 (1993).

56. Pasaoglu, H., Sancak, B. \& Bukan, N. Lipid peroxidation and resistance to oxidation in patients with type 2 diabetes mellitus. Tohoku J. Exp. Med. 203 211-218 (2004) 